\title{
DESCRIPTION D'UNE NOUVELLE ESPÈCE DU GENRE
}

\section{METATHELAZIA (NEMATODA-SPIRUROIDEA)}

\author{
Par Ettore Blocc A et Alain G. CHABAUD
}

Le genre Metathelazia, au moment où il a été isolé par Skinker (1931), ne comprenait qu'un très petit nombre d'espèces. Depuis cette époque, de nombreuses espèces nouvelles ont été décrites et le genre a pris un grand intérêt, car, bien qu'ayant un certain nombre de caractères communs, les espèces sont remarquables par leur extrême diversité. Nous décrirons ici une nouvelle espèce qui, en raison de son aspect gracile, sera désignée sous le nom de $M$. exilis.

Matériel. - Une femelle, trois mâles entiers et plusieurs fragments des deux sexes ont été récoltés dans les bronchioles d'un spécimen d'Herpestes caffer (Gmelin 1788), capturé en Erythrée et mort peu après son arrivée au Jardin Zoologique de Rome. (Le matériel est déposé dans les collections de !'Institut de Parasitologie de la Faculté de Médecine de Paris).

Description. - Corps très fin dans les deux sexes, ayant l'aspect macroscopique d'une filaire. La cuticule, recouverte d'un « téguminal sheath », est très fine ; elle est dépourvue de stries transversales, mais présente des petits tubercules.

La tête (fig. 1, A) est ornée de six grands lobes charnus (deux latéraux et quatre submédians) ; le grand développement des lobes latéraux la rend spécialement proche de $M$. cesophagea. Le cycle externe de papilles céphaliques comprend deux grosses paires latéro-médianes et deux paires médio-médianes plus petites; le cycle interne n'est pas distinguable. Il est remarquable qu'en l'absence de papilles internes, les paires médio-médianes soient en position plus antérieure que chez les espèces voisines pourvues d'un cycle interne bien développé. Les deux grosses amphides ont une situation légèrement plus antérieure que les papilles latéromédianes.

La limite entre les portions musculaire et glandulaire de l'œesophage n'est pas très nettement tranchée. L'intestin, très dilaté dans

ann. de Parasitologie, T. XXVII, $\mathrm{N}^{\circ} 4 .-1952$. 
sa portion antérieure, surtout chez la femelle, ne présente pas cependant de cæca distincts. L'anneau nerveux entoure le tiers postérieur de l'intestin musculaire (fig. 1, B, C) ; le pore excréteur est situé au même niveau que les diérides, nettement en arrière de l'anneau nerveux. Cette région est occupée par de gros ganglions qui existent chez tous les nématodes, mais se voient rarement avec une telle

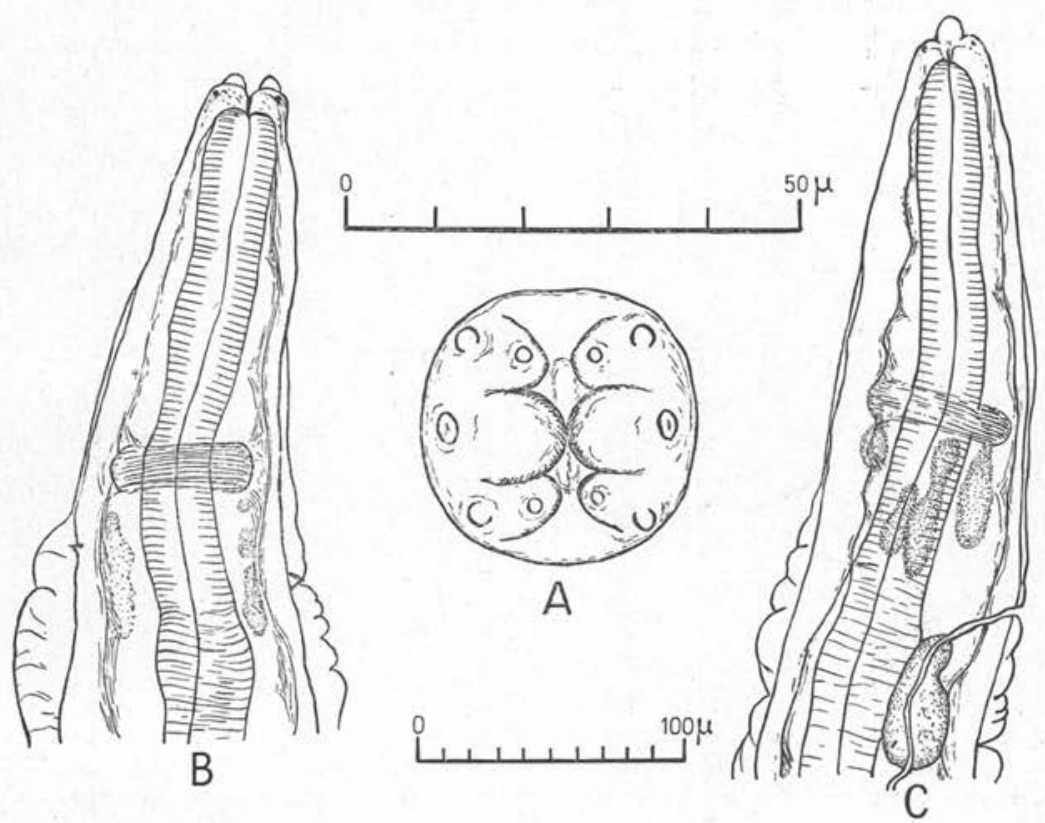

Fig. 1. - M. exilis. Femelle. A. Extrémité céphalique; vue apicale (échelle : $0-50 \mu$ ). B. Extrémité antérieure; vue médiane (échelle: $0-100 \mu$ ). C. Extrémité antérieure; vue latérale (échelle : $0-100 \mu$ ).

netteté chez les adultes. L'appareil excréteur a la même anatomie que dans les espèces voisines.

Mâle. - Long de $10,25 \mathrm{~mm}$., la largeur maxima du corps, en faisant abstraction du «teguminal sheath », est de $110 \mu$. Csophage musculaire long de $190 \mu$, et œesophage glandulaire long de $170 \mu$; milieu de l'anneau nerveux à $115 \mu$ de l'extrémité antérieure ; diérides symétriques et pore excréteur au même niveau à $160 \mu$ de l'extrémité antérieure. La queue (fig. $2, \mathrm{~A})$, très courte $(45 \mu)$, possède une face ventrale qui est légèrement différenciée pour former un plateau triangulaire plan (fig. 2, B). Il existe 23 papilles génitales et 2 phasmides réparties de la facon suivante : un groupe antérieur comprend 
quatre paires pré-cloacales largement espacées ; un groupe péricloacal comprend une papille impaire antérieure, deux paires latérales grosses et deux paires subventrales un peu plus petites ; un groupe postérieur comprend une paire latérale assez grande, une paire subventrale plus petite qui est située au même niveau, et une dernière paire, petite, et plus postérieure. Les phasmides sont situées symétriquement, en arrière de la paire la plus postérieure. Les spicules, subégaux, sont longs de $130-150 \mu$ et ont lanatomie habi-

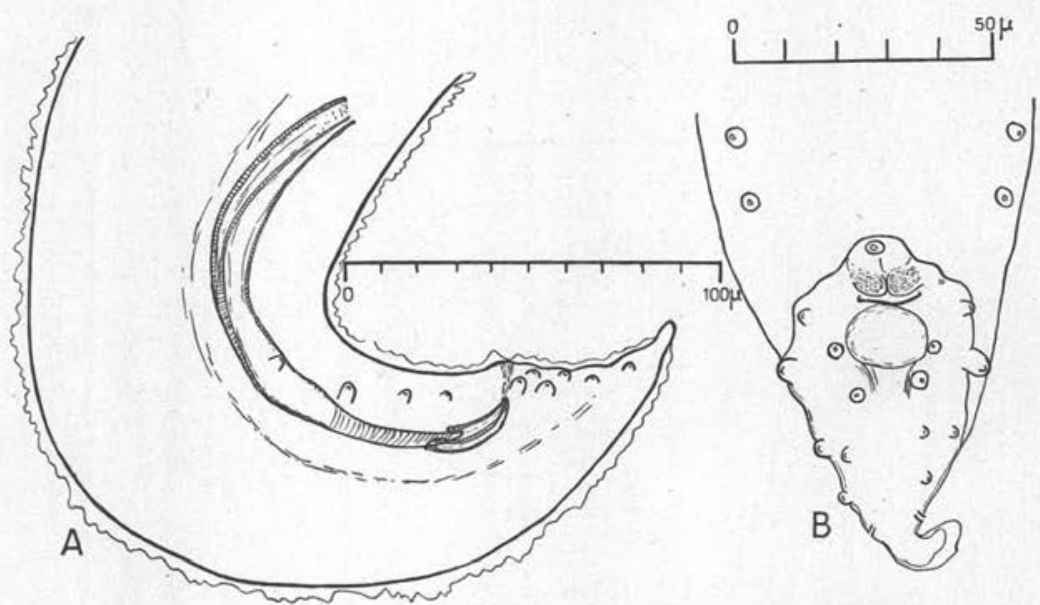

Fıg. 2. - M. exilis. Mâle. A. Extrémité postérieure ; vue latérale (Le spicule gauche et la moitié gauche du gubernaculum n'ont pas été représentés). B. Pointe caudale; vue ventrale (La pointe distale du gubernaculum apparait au-dessus du cloaque).

tuelle aux espèces du genre (manche proximal long de $110 \mu$ et pointe distale longue de $35 \mu$ ). Le gubernaculum est formé de deux pièces égales et indépendantes l'une de l'autre ; chacune, longue de 25-20 $\mu$, a la forme d'une gouttière, dans la concavité de laquelle glisse le spicule correspondant.

Femelle. - Longue de $29 \mathrm{~mm}$. et large de $150 \mu$. L'osophage musculaire et l'œsophage glandulaire mesurent respectivement 190 et $270 \mu$. L'anneau nerveux, les diérides et le pore excréteur sont respectivement à 140,190 et $195 \mu$ de l'apex.

L'extrémité postérieure (fig. 3, A), brusquement tronquée à partir de l'orifice vulvaire, est déportée dorsalement. La vulve est située à environ $130 \mu$ de la pointe caudale et l'anus s'ouvre à mi-distance, à $65 \mu$ de la pointe caudale. L'ovéjecteur (fig. $3, \mathrm{C}$ ) a une anatomie 
comparable à celle des Spirurides les plus primitifs. Le vestibule, long de $1,7 \mathrm{~mm}$., est fermé par deux sphincters ; l'un pré-vulvaire (fig. $3, \mathrm{~A}$ ), d'un diamètre d'environ $50 \mu$, a une forme presque sphérique ; l'autre (fig. $3, \mathrm{C}$ ), qui forme la limite entre le vestibule et la trompe, a une structure plus complexe ; il a la forme d'un conduit long de $300 \mu$ et large d'environ $45 \mu$ et se trouve renforcé à la limite

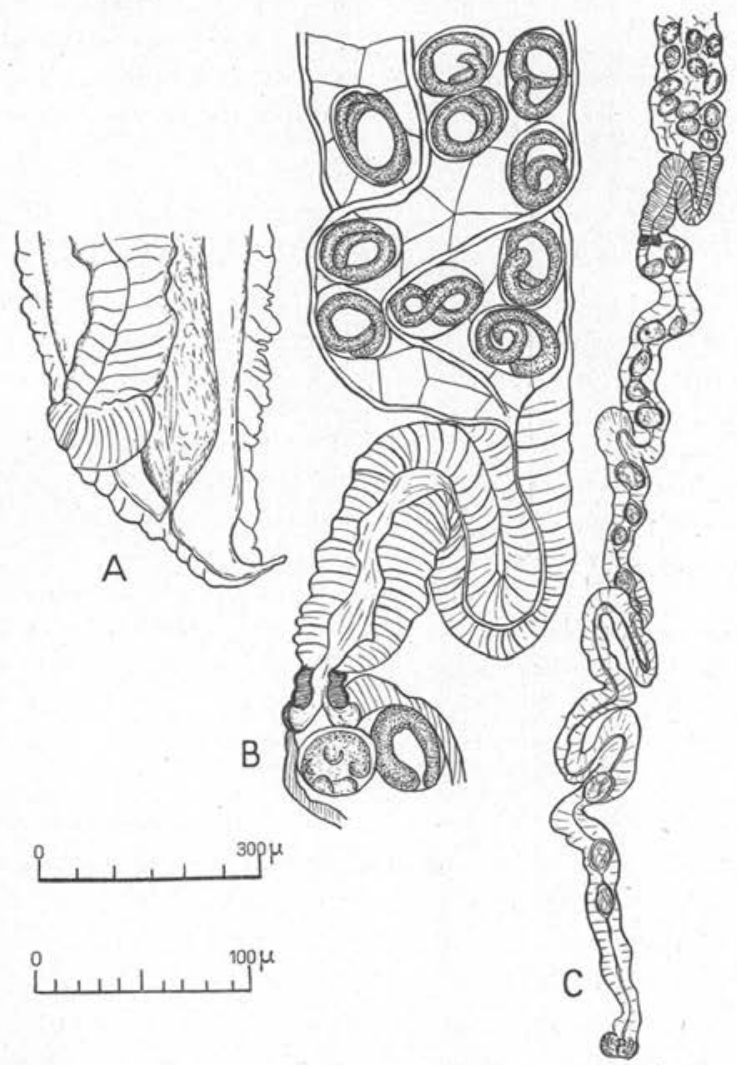

Fig. 3. - M. exilis. Femelle. A. Extrémité postérieure ; vue latérale. B. Détail du sphincter limitant la trompe et le vestibule. C. Dissection de l'ovejecteur. (A-B : échelle $0-100 \mu ; \mathrm{C}$ : échelle $0-300 \mu$ ).

du vestibule par un étranglement annulaire haut de $20 \mu$. La trompe est atrophiée et les deux utérus sont raccordés presque directement au sphincter. Eufs embryonnés longs de $42 \mu$ et larges de $32 \mu$.

Discussion. - Les différentes espèces du genre sont très faciles à reconnaitre entre elles et il suffira, pour préciser la diagnose, de $M$, exilis, de compléter le tableau dichotomique présenté par Ge- 
richter (1948) dans le premier paragraphe qui concerne les espèces pourvues de «six conspicuous perioral lip-like projections 》 (1),

1. (2) Esophage avant plus du $1 / 7^{\circ}$ de la longueur du corps.

Paires de papilles génitales du type $1+1+7=9$; distance vulve-anus $=$ distance anus-pointe caudale ; longueur du vagin $=1 \mathrm{~mm} . ;$ longueur des spicules $=220 \mu \ldots \ldots \ldots \ldots$. ...................... osophagea, Gerichter, 1948.

2. (1) Esophage ayant moins du $1 / 10^{\circ}$ de la longueur du corps.

3. (4) Distance vulve-anus environ double de la distance anus-pointe caudale.

Papilles génitales du type $3+6=9$. Spicules longs de $375 \mu$; vagin long de $1 \mathrm{~mm}$.

M. servalis, Chabaud et Biocca, 1950 (2).

4. (3) Distance vulve-anus $=$ distance anus-pointe caudale.

5. (6) Largeur du corps de la femelle inférieure à $200 \mu$.

Vagin long de $2 \mathrm{~mm}$. ; spicules longs de $140 \mu$; papilles génitales du type $4+1+6=11 . \ldots \ldots \ldots \ldots . M$. exilis, sp. nov.

6. (5) Largeur du corps de la femelle supérieure à $400 \mu$.

7. (8) Longueur du corps de la femelle supérieure à $26 \mathrm{~mm}$. Vagin long de $7 \mathrm{~mm}$. ; spicules longs de $200 \mu$; papilles génitales du type

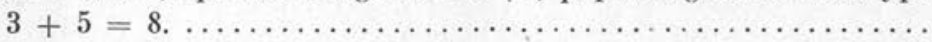

8. (7) Longueur du corps de la femelle inférieure à $16 \mathrm{~mm}$. Vagin long de $800 \mu$; spicules longs de $220 \mu$; papilles génitales du type $4 / 7+4=8 / 11$. M. ascaroides (V. Linstow, 1879), Dougherty, 1943.

L'étude générale des Spirurida révèle que l'anatomie céphalique a une très grande importance phylogénique et que, au contraire, certains caractères tels que la structure cuticulaire ou la forme des spicules varient largement dans des groupes proches. Le principal intérêt du genre Metathelazia réside dans le fait qu'il existe ici, au contraire, une grande diversité dans la structure céphalique qui s'oppose à une certaine uniformité dans quelques éléments tels que la cuticule, les spicules ou le gubernaculum. Nous pensons qu'une telle anomalie ne peut se rencontrer que dans un groupe polyphylétique où les phénomènes de convergence ont joué de façon essentielle. La création d'une nouvelle sous-famille, les Vogeloidinæ et la coupure en différents genres annoncée par Dougherty (1951), nous

(1) M. hainanensis (Wu et $\mathrm{Hu}, 1938$ ) doit être exclu de ce paragraphe et rapproché de M. capsulata Gerichter, 1948 (Dougherty, Proc. Helm. Soc. Wash., sous presse).

(2) M. zorillæ (Seurat, 1915) Chabaud, 1949, paraît proche de cette espèce par la structure céphalique et par la position des diérides, mais l'espèce n'est connue qu'à l'état larvaire et ne peut figurer dans ce tableau. 
paraissent donc très utiles et nous partageons entièrement le point de vue de cet auteur lorsqu'il considère les Metathelazia comme un groupe indépendant probablement archaïque.

Nous ne pouvons cependant pas suivre Dougherty lorsqu'il place cette nouvelle sous-famille parmi les Metastrongylidær. On négligerait ainsi les éléments essentiels (structure céphalique, œesophage...) pour ne considérer que des éléments qui, dans l'évolution générale des Spiruridr, apparaissent spécialement sensibles aux phénomènes de convergence tels que la cuticule (existence d'un "teguminal sheath ») ou la forme du gubernaculum.

RÉsumé. - Description d'une nouvelle espèce du genre Metathela$z i a$. Nous exposons les faits qui nous incitent à placer ce genre parmi les Spiruroidea et non parmi les Metastrongylidæ.

\section{BiBLIOGRAPHIE}

Chabaud (A.-G.). - Contribution à l'étude du cycle évolutif du genre Metathelazia Skinker, 1931. Ann. Parasit., XXIX, 1949, 60-66.

- et Brocca (E.). - Description de Metathelazia servalis, n. sp., et observation sur le genre Metathelazia. Bull. Soc. Zool. France, LXXV, 1950, 260267.

Davtian (E. A.). - Ein neuer Nematoden aus den Lungen der Hauskatze, Osleroides massino, nov. sp. Deutsch. Tierarztl. Wochenschr., XLI, 1933, 372-374 (1).

Dougherty (E. C.). - The genus Filaroides van Beneden, 1858, and its relatives : Preliminary note. Proc. Helminth. Soc. Wash., X, 1943, 69-74.

Evolution of Zoöparasitic groups in the phylum Nematoda, with special reference to host-distribution. J. Parasit., XXXVII, 1951, 353-378.

Gerichter (C. B.). - Three new species of the genus Metathelazia (Nematoda). J. Parasit., XXXIV, 1948, 75-83.

v. Linstow (O. F. B.). - Helminthologische Untersuchungen. Jahresh. Ver. f. vaterlünd. Naturk. Würtenberg, XXXV, 1879, 313-342 (1).

Seurat (L.-G.). - Contributions nouvelles à l'étude des formes larvaires des nématodes parasites hétéroxènes. Bull. Biol. France et Belg., LII, 1919, 344-378.

(1) Ce travail n'a pas été consulté.

Institut de Parasitologie de la Faculté de Médecine de Rome (Directeur : E. Biocca)

et Institut de Parasitologie de la Faculté de Médecine de Paris (Directeur : H. Galliard) 